\title{
Interplay between coarsening and nucleation in an Ising model with dipolar interactions
}

\author{
Sergio A. Cannas, ${ }^{1, *}$ Mateus F. Michelon, ${ }^{2, \dagger}$ Daniel A. Stariolo, ${ }^{3, \ddagger}$ and Francisco A. Tamarit ${ }^{4,}$ \\ ${ }^{1}$ Instituto de Física de la Facultad de Matemática, Astronomía y Física (IFFAMAF-CONICET), Universidad Nacional de Córdoba, \\ Ciudad Universitaria, 5000 Córdoba, Argentina \\ ${ }^{2}$ Instituto de Física "Gleb Wataghin," Caixa Postal 6165, Universidade Estadual de Campinas-UNICAMP, \\ 13083-970, Campinas, São Paulo, Brazil \\ ${ }^{3}$ Departamento de Física, Universidade Federal do Rio Grande do Sul, Caixa Postal 15051, 91501-970 Porto Alegre, \\ Rio Grande do Sul, Brazil \\ ${ }^{4}$ Instituto de Física de la Facultad de Matemática, Astronomía y Física (IFFAMAF-CONICET), Universidad Nacional de Córdoba, \\ Ciudad Universitaria, 5000 Córdoba, Argentina
}

(Received 29 April 2008; revised manuscript received 9 October 2008; published 14 November 2008)

\begin{abstract}
We study the dynamical behavior of a square lattice Ising model with exchange and dipolar interactions by means of Monte Carlo simulations. After a sudden quench to low temperatures, we find that the system may undergo a coarsening process where stripe phases with different orientations compete, or alternatively it can relax initially to a metastable nematic phase and then decay to the equilibrium stripe phase through nucleation. We measure the distribution of equilibration times for both processes and compute their relative probability of occurrence as a function of temperature and system size. This peculiar relaxation mechanism is due to the strong metastability of the nematic phase, which goes deep into the low-temperature stripe phase. We also measure quasiequilibrium autocorrelations in a wide range of temperatures. They show a distinct decay to a plateau that we identify as due to a finite fraction of frozen spins in the nematic phase. We find indications that the plateau is a finite-size effect. Relaxation times as a function of temperature in the metastable region show super-Arrhenius behavior, suggesting a possible glassy behavior of the system at low temperatures.
\end{abstract}

DOI: 10.1103/PhysRevE.78.051602 PACS number(s): 64.60.qe, 64.75.St, 75.40.Gb, 75.40.Mg

\section{INTRODUCTION}

It is well known that the usual long-range order characteristic of a ferromagnetic phase dominated by exchange interactions can be destroyed by the presence of dipolar interactions inducing the formation of magnetic domains [1]. In some cases, typically when perpendicular anisotropy is present, the dipolar interactions are antiferromagnetic in character and compete with the ferromagnetic exchange interactions [2,3], giving rise to antiferromagnetic or stripe phases. These phases are common in ferromagnetic thin films with perpendicular anisotropy and have been the subject of intense experimental [4-8], theoretical [9-11], and numerical [12-17] work in the last 20 years. In the strong anisotropy limit, an ultrathin film can be modeled by a system of magnetic dipoles on a square lattice in which the magnetic moments are oriented perpendicular to the plane of the lattice, with both nearest-neighbor ferromagnetic exchange interactions and long-range dipole-dipole interactions between moments. The thermodynamics of this system is ruled by the dimensionless Ising Hamiltonian:

$$
\mathcal{H}=-\delta \sum_{\langle i, j\rangle} \sigma_{i} \sigma_{j}+\sum_{(i, j)} \frac{\sigma_{i} \sigma_{j}}{r_{i j}^{3}},
$$

where $\left\{\sigma_{i}= \pm 1, i=1, \ldots, N\right\}$ are Ising spins and $\delta$ stands for the ratio between the exchange $J_{0}>0$ and the dipolar

\footnotetext{
*cannas@famaf.unc.edu.ar

†michelon@ifi.unicamp.br

†stariolo@if.ufrgs.br

§tamarit@famaf.unc.edu.ar
}

$J_{d}>0$ interaction parameters, i.e., $\delta=J_{0} / J_{d}$. The first sum runs over all pairs of nearest-neighbor spins and the second one over all distinct pairs of spins of the lattice; $r_{i j}$ is the distance, measured in crystal units, between sites $i$ and $j$. The energy is measured in units of $J_{d}$. The equilibrium phase diagram of this system has been extensively studied $[12,13,15,16]$, while several dynamical properties at low temperatures were studied in [18-21]. The threshold for the appearance of the stripe phase in this model is $\delta_{c}=0.425$ $[12,16]$. For $\delta>\delta_{c}$ the system presents a sequence of striped ground states, characterized by a constant width $h$, whose value increases exponentially with $\delta[3,12]$.

In [13] it was shown that in the range $1 \leqslant \delta \leqslant 3$ the system presents a first-order phase transition between a lowtemperature stripe phase with broken translational and rotational symmetry and a high-temperature tetragonal liquid phase. The tetragonal phase is a disordered state characterized by a fourfold discrete rotational symmetry [22]. In a subsequent work [14] it was shown that for a narrow window around $\delta=2$ the model shows an intermediate nematic phase, between the stripe and tetragonal ones, where the system has short-range positional order but long-range orientational order. The transition from the tetragonal to either the nematic or the striped state can be well characterized by an orientational order parameter introduced by Booth et al. [22]. Such an order parameter is not appropriate to characterize the transition between the nematic and the striped states, because both phases present orientational order. Instead of that, it was shown in Ref. [14] that the transition can be well characterized through the scaling properties of the static structure factor. Further details will be presented in Sec. III A.

The nematic phase exists between two critical temperatures $T_{1}<T<T_{2}$. For finite sizes these are actually pseudo- 

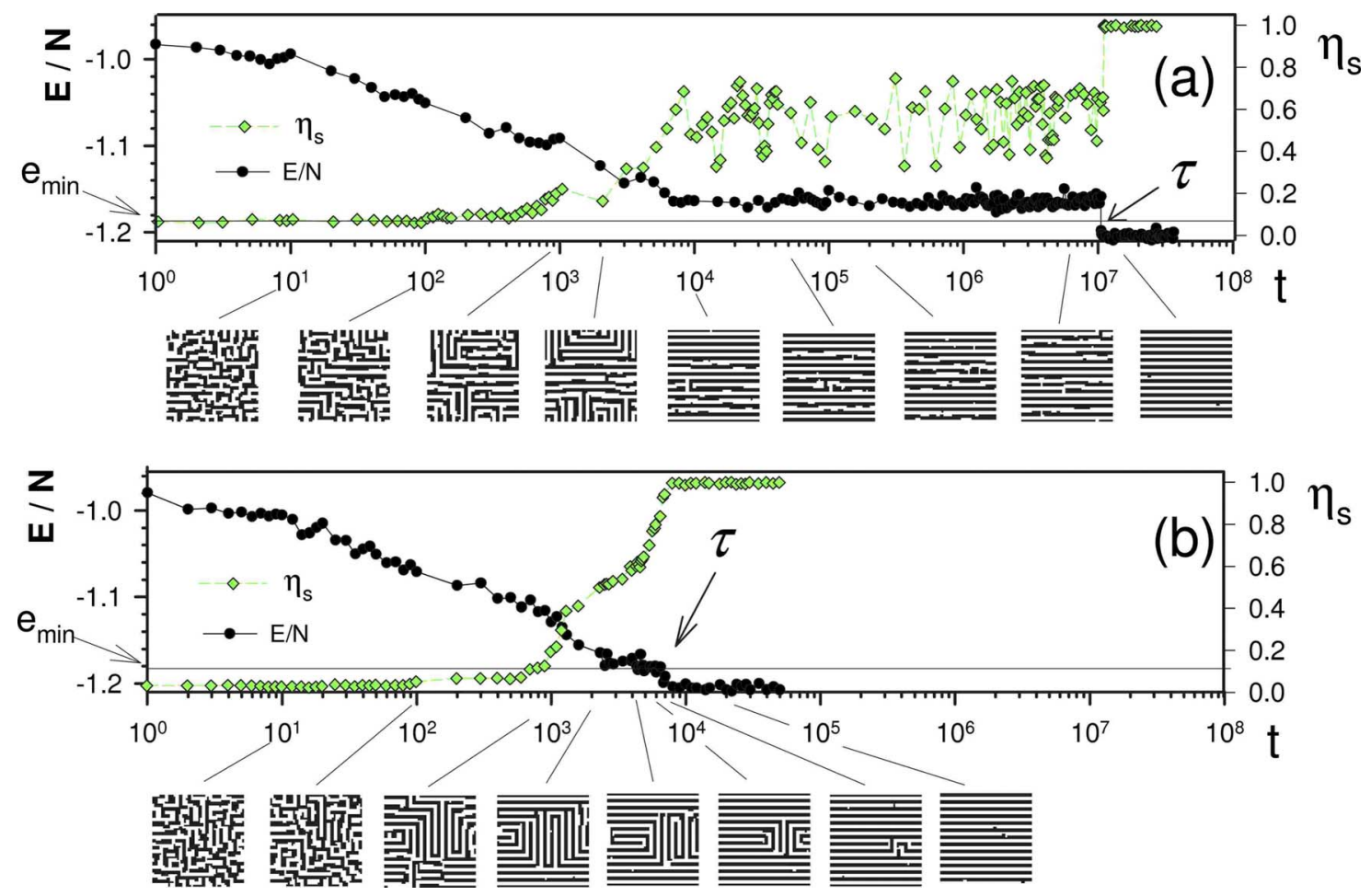

FIG. 1. (Color online) Time evolution of the stripe order parameter and energy per spin in a single MC run $(\delta=2, L=52$, and $T=0.65)$ for two different realizations of the stochastic noise. Some snapshots of the spin configurations at different times are shown below the figures. (a) Competition between nematic domains (coarsening), which leads to a metastable nematic state, followed by an activated relaxation to the striped state (equilibration through nucleation); (b) competition between nematic and striped domains (coarsening) which leads directly to equilibration.

critical temperatures $T_{1}(L)$ and $T_{2}(L)$, with finite energy barriers between phases. The extrapolation to the thermodynamic limit gives $T_{1}(\infty) \approx 0.772$ and $T_{2}(\infty) \approx 0.797$ [14]. For $L \sim 50(L=48-56)$ we can estimate $T_{1}(L)$ and $T_{2}(L)$ as the averages between the positions of the minimum of the cumulant and the maximum of the specific heat, so $T_{1}(50) \approx 0.78$ and $T_{2}(50) \approx 0.81$. For the range of system sizes considered $(L \sim 50)$, the energy barriers between the nematic and the tetragonal phases are rather small, while the barriers between the stripe and the nematic phases are very large [14]. Hence, under a cooling from high temperature no metastable tetragonal states are observed, but we see a strong metastability in the nematic phase. This can be observed in Fig. 19 of Ref. [14]. Although metastability strongly resembles a first-order phase transition, we were not able to determine the nature of the stripe-nematic transition unambiguously: while the nematic order parameter presents a jump at $T_{1}$ and the free energy barriers seem to diverge in the thermodynamic limit, the internal energy is continuous at the transition and the peak in the specific heat tends to saturate [14]. Further Monte Carlo (MC) simulations showed that the nematic phase is present also for other values of $\delta \neq 2$ in different parts of the phase diagram, mainly around the phase borders between striped states of different widths [16].

Here we study the out-of-equilibrium dynamical properties of the two-dimensional Ising model with exchange and dipolar interactions described by the Hamiltonian (1) with $\delta=2$. We performed quenches of the system from initial conditions at high temperatures directly to the stripe phase $\left(T_{f}\right.$ $\leqslant T_{1}$ ). Our main result is the identification of two kinds of process: a slow coarsening toward a metastable nematic phase followed by nucleation of the stripe phase in a background of the metastable nematic and also direct coarsening of the stripe phase without an intermediate nucleation process (see Fig. 1). In each realization the system chooses one of both paths to the final stripe phase with probabilities that depend on the final temperature of the quench. We computed these probabilities from Monte Carlo simulations and then using arguments from homogeneous nucleation theory, finite-size-scaling analysis, and known growth laws for critical dynamics we were able to characterize completely both kinds of processes in the whole temperature range below the stripe-nematic transition.

We also made slow cooling experiments, similar to those already discussed in [14], and which show strong hysteretic behavior in the energy. We confirm that hysteresis is associated with a strong metastability of the nematic phase, while the tetragonal phase shows negligible metastability in the energy under cooling. Finally, (quasi)equilibrium autocorrelation functions obtained for a whole range of temperatures between the tetragonal and the stripe phases show slow relaxation characterized by stretched exponential decay. In the (metastable) nematic phase the relaxation times obtained from the autocorrelations exhibit a strong growth which can be well fitted by a Vogel-Fulcher-Tamman relation, similar to the behavior of fragile glass formers, with a relatively high $\left(T_{0} \simeq 0.45\right)$ divergence temperature.

In Sec. II we describe the quench experiments and the associated phenomenology. In Sec. III the slow cooling ex- 
periments and the behavior of the equilibrium correlations are discussed. In Sec. IV we present a discussion of results and brief conclusions.

\section{RELAXATION AFTER A QUENCH FROM HIGH TEMPERATURE: INTERPLAY BETWEEN COARSENING AND NUCLEATION}

We performed quenches from infinite temperature (completely random initial configuration) down to temperatures $T<T_{1}$. We also compared with the case where the initial configuration is taken from an equilibrated state at temperatures $T \sim 1$. The results were the same.

Growing order in the system can be characterized in several ways. A natural one is to define a stripe order parameter through the static structure factor

$$
S(\vec{k}) \equiv\left\langle\left|\hat{\sigma}_{\vec{k}}\right|^{2}\right\rangle=\frac{1}{N^{2}} \sum_{\vec{r}} \sum_{\vec{r}^{\prime}} C\left(\vec{r}, \vec{r}^{\prime}\right) e^{i \vec{k} \cdot\left(\vec{r}-\vec{r}^{\prime}\right)}
$$

where $\hat{\sigma}_{\vec{k}}=(1 / N) \sum_{\vec{r}} e^{i \vec{k} \cdot \vec{r}} \sigma_{\vec{r}}$ is the discrete Fourier transform of $\sigma_{\vec{r}}$. It can be shown that in a pure striped state of width $h$ the only nonzero components correspond to $\left(k_{x}, k_{y}\right)$ $=(0, \pm \pi p / h)$ (horizontal stripes) or $\left(k_{x}, k_{y}\right)=( \pm \pi p / h, 0)$ (vertical stripes), where $p$ takes all the odd integer values between 1 and $h$. In the present study the ground state has $h=2$. Since all the phases we are dealing with in this case present a discrete rotational symmetry, of either $90^{\circ}$ or $180^{\circ}$ with respect to the coordinate axes, the maxima of $S(\vec{k})$ will be located at $\left(k_{x}, k_{y}\right)=\left( \pm k_{0}, 0\right)$ and/or $\left(k_{x}, k_{y}\right)=\left(0, \pm k_{0}\right)$, with $k_{0}$ some value close to $\pi / 2$. Then we can define a stripe order parameter as

$$
\eta_{s}=2\left[S\left(k_{0}, 0\right)+S\left(0, k_{0}\right)\right] .
$$

This order parameter will take values close to 1 for states with long-range $h=2$ stripe order, and zero (in the thermodynamic limit) for any state without long-range positional order. In particular, in a nematic phase it will approach zero as $1 / L$ (see Sec. III A).

The typical relaxation behavior of the system can be appreciated in Fig. 1, where we plotted the time evolution of the stripe order parameter $\eta_{s}$ and the instantaneous energy per spin $E(t) / N$, for two different realizations of the stochastic noise, together with typical spin configurations along the evolution. We see that the system can relax through two different types of mechanisms. In Fig. 1(a) the system relaxes first into a metastable nematic state (in Sec. III A we will analyze such a state in detail), where it stays during a long time $\tau$, after which it suddenly relaxes to the equilibrium stripe state. In other words, there is a two-step relaxation: first a coarsening process, where domains of the nematic phase compete (horizontal and vertical stripe orientations), followed by a nucleation of the stable phase. The clear jump in the order parameter is an indication that the final decay to the stripe equilibrium configuration is a result of a nucleation process (we will verify this quantitatively later) and not a mere adjustment of stripe width. This last possibility could be due to an incommensurability of the ground state with the lattice size, but in this case the order parameter would not

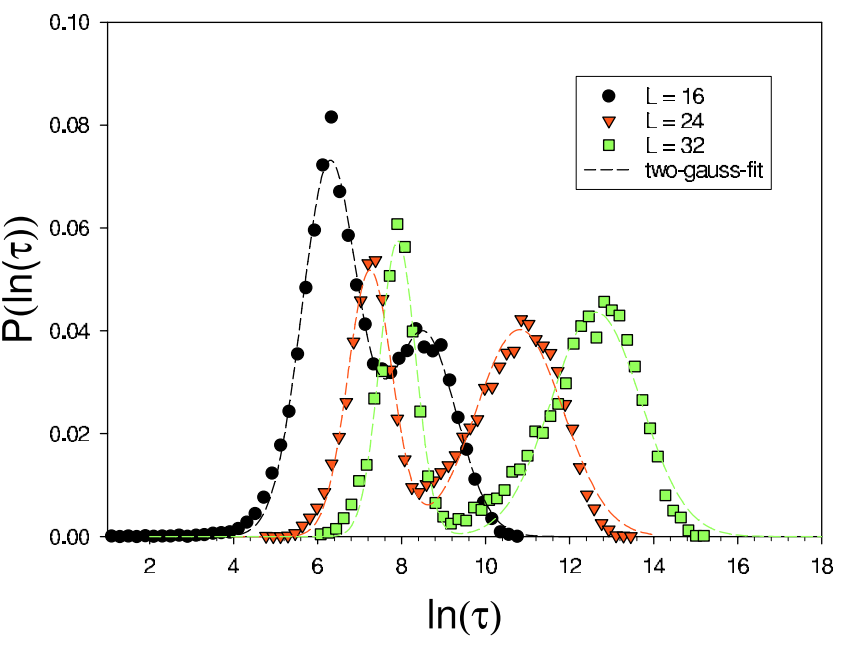

FIG. 2. (Color online) Normalized frequency $P(\tau)$ vs $\ln \tau$ for $\delta=2, T=0.65$, and different system sizes. The lines correspond to a fitting with a superposition of two log-normal distributions. Sample size for the histograms was $\sim 2 \times 10^{4}$.

show such strongly discontinuous behavior. In Fig. 1(b) the system forms domains of both the stable (striped) and metastable (nematic) phases that compete and relax directly to the equilibrium state through a coarsening process. Both types of relaxation process can happen with a certain probability which depends on the temperature. To analyze these processes quantitatively we computed the equilibration time probability distribution.

The equilibration time $\tau$ is defined as the time [in Monte Carlo steps (MCS)] that the system takes to reach the stable, crystal-like striped state after a quench. The criterion to determine $\tau$ was the following. For each quench temperature $T<T_{1}$ we calculated the equilibrium energy per spin histogram along a large single MC path starting from the ground state. This histogram presents two Gaussian-shaped peaks, each one centered at the averages $u_{\text {str }}(T)$ and $u_{d i s}(T)$ [14]. Between the two peaks the histogram presents a finite minimum (at least for a finite system), associated with the free energy barrier that separates both phases. We also measured the standard deviation of the low energy peak associated with the striped phase $\sigma_{\text {str }}(T)$ and defined $e_{\min }(T)=u_{\text {str }}+\sigma_{\text {str }}$, verifying that it is well below the central minimum of the histogram; $e_{\min }$ is almost independent of the system size for $L \geqslant 32$, so it can be easily calculated using small system sizes. Then for each quench we calculated the average energy per spin $e(t) \equiv\langle H\rangle / N$. When $e(t)$ fell below $e_{\min }$ we stopped the simulation, defining $e(\tau)=e_{\min }$ (see Fig. 1). Repeating this procedure, we obtained the probability distribution (normalized frequency) $P(\tau)$ of the stochastic variable $\tau$ for different values of $T$ and $L$.

The above-mentioned relaxation mechanisms are reflected in a characteristic two-peak structure in $P(\tau)$, each one centered at typical values corresponding to very different time scales, as shown in Figs. 2 and 3. This probability distribution can be very well fitted using a superposition of two $\log$-normal functions (see Figs. 2 and 3) $P(\ln \tau)=P_{1}(\ln \tau)$ $+P_{2}(\ln \tau)$ with 


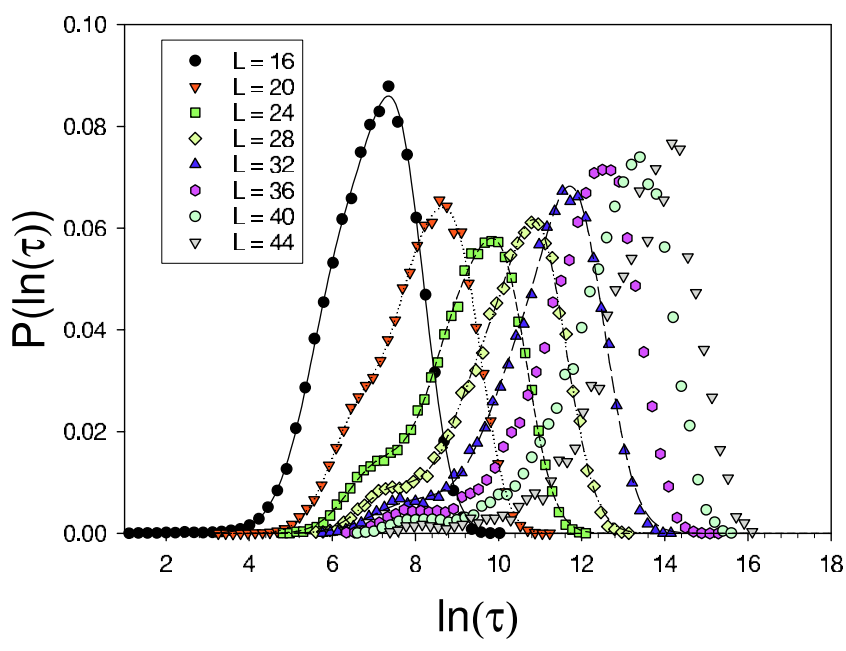

FIG. 3. (Color online) Normalized frequency $P(\tau)$ vs $\ln \tau$ for $\delta=2, T=0.77$, and different system sizes. The lines correspond to a fitting with a superposition of two log-normal distributions Eqs. (4). Sample sizes for the histograms run between $\sim 4 \times 10^{3}(L=44)$ and $\sim 5 \times 10^{4}(L=16)$.

$$
P_{i}(\ln \tau)=A_{i} \exp \left(\frac{\left(\ln \tau-\mu_{i}\right)^{2}}{2 \sigma_{i}^{2}}\right), \quad i=1,2,
$$

where $A_{i}$ are normalization constants. Using these fittings we can see in Fig. 4 that the characteristic time scales of both peaks increase as $T$ decreases.

These fittings also allowed us to estimate the averages $\langle\tau\rangle_{i}=\exp \left(\mu_{i}+\sigma_{i}^{2} / 2\right), i=1,2$, associated with each process. Both averages increase with $L$, so we can use finite-sizescaling analysis to determine the nature of the associated processes.

\section{Finite-size scaling of the equilibration times}

Suppose that the relaxation is completely governed by a competition between ground state domains, that is, by a

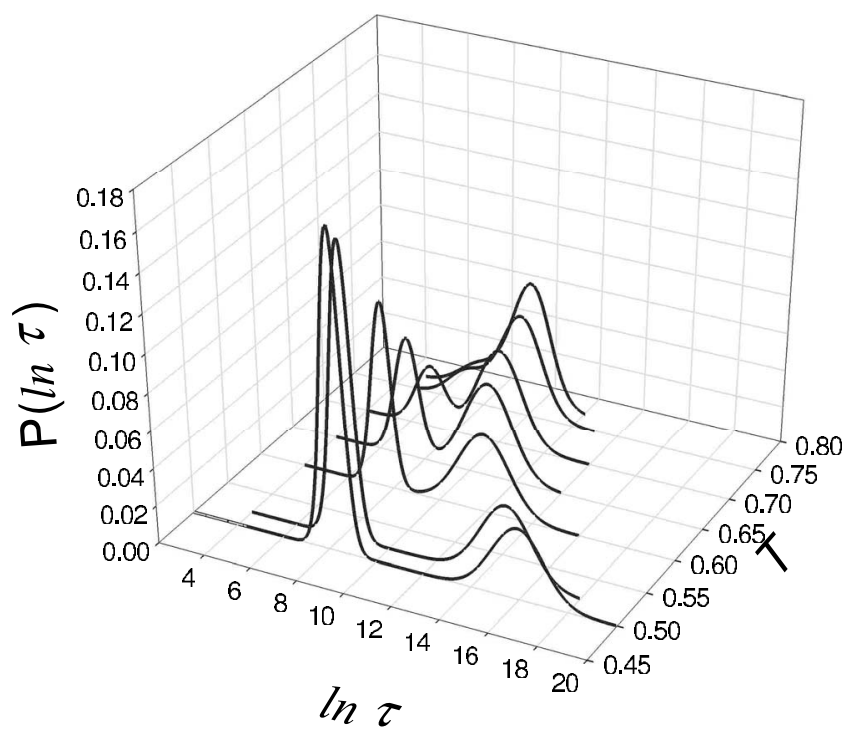

FIG. 4. Fitted probability distribution $P(\tau)$ as a function of $\ln \tau$ and $T$ for $\delta=2$ and $L=24$.

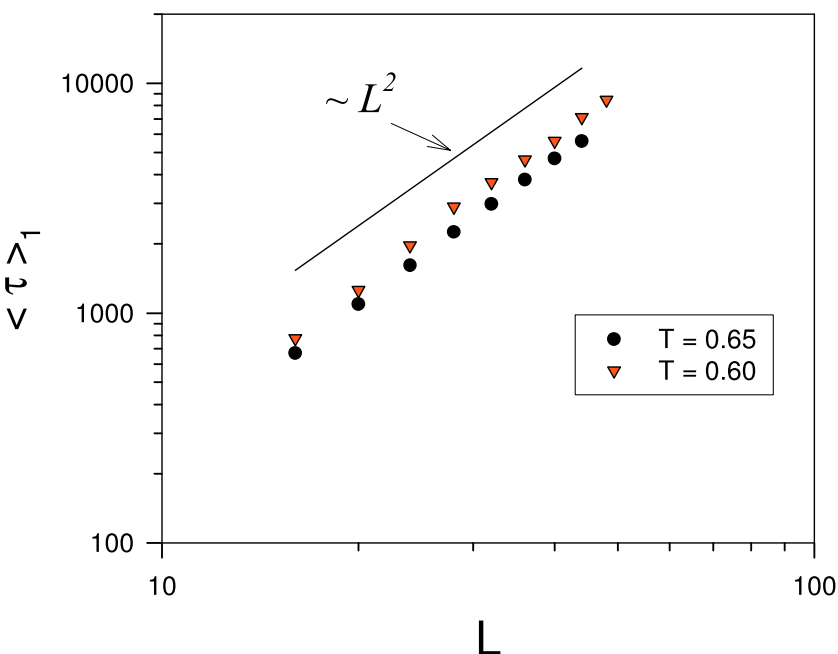

FIG. 5. (Color online) $\langle\tau\rangle_{1}$ vs $L$ from two log-normal fittings Eqs. (4).

curvature-driven relaxation of the domain walls. The excess of energy with respect to the ground state is given by $\delta e$ $=\gamma n, \gamma$ being the "surface" tension per unit length of the domain walls and $n$ the fraction of spins belonging to them; $n$ is approximately given by $n \simeq b N_{d}(t) l(t) / N$, where $N_{d}(t)$ is the average number of domains, $l(t)$ is the average linear size of the domains, and $b$ is a geometrical factor. Using $N_{d}$ $\propto N / l^{2}(t)$ and grouping all these factors, we get $\delta e \sim l^{-1}(t)$. For $\delta=2$ the energy distribution of the striped phase is very narrow and lies very near the ground state, so the criterion $e(\tau)=e_{\min }$ implies $\delta e \ll 1$, a condition that is attained when $l(\tau) \sim L$. Since for a heat bath dynamics with nonconserved order parameter (class $A$ system) we can assume $l(t) \sim t^{1 / 2}$, we expect the scaling $\langle\tau\rangle \sim L^{2}$. We verified that $\langle\tau\rangle_{1} \sim L^{2}$ for a wide range of values of $T$ and $L$ (see an example in Fig. 5), thus confirming that $P_{1}(\tau)$ is associated with a simple coarsening process.

Now suppose that relaxation is governed by an homogeneous nucleation process. According to classical nucleation theory $\langle\tau\rangle$ follows approximately an Arrhenius law, $\langle\tau\rangle$ $=\tau_{0} \exp [\Delta(L, T) / T], \Delta(L, T)$ being the height of the free energy barrier to nucleation. In a finite two-dimensional system [23] $\Delta\left(T_{m}\right) \sim L, T_{m}$ being the melting temperature; in our case $T_{m}=T_{1}$. In an infinite system below the melting point, classical nucleation theory predicts that $\Delta(T) \equiv \Delta(\infty, T)$ is given by the excess of free energy of a critical droplet with average linear size $\xi(T)$ of the ordered (striped) phase inside the disordered (nematic) metastable state. Thus, if $L \gg \xi(T)$ the free energy barrier is expected to be almost independent of the system size when $T<T_{m}$. But, if $L<\xi(T)$, the droplet never reaches the critical size and the excess of free energy is dominated by surface tension. Hence, the transition to the crystal phase will occur when the crystal droplet reaches the system size and therefore we expect $\Delta(L, T) \sim L$ and $\ln \langle\tau\rangle$ $\sim L / T$.

Figure 6 shows that $\langle\tau\rangle_{2}$ follows such scaling, suggesting its identification with the average nucleation time $\tau_{\text {nucl }}$. However, due to the procedure we used to obtain it, $\langle\tau\rangle_{2}$ actually corresponds to the crystallization time, which is not neces- 


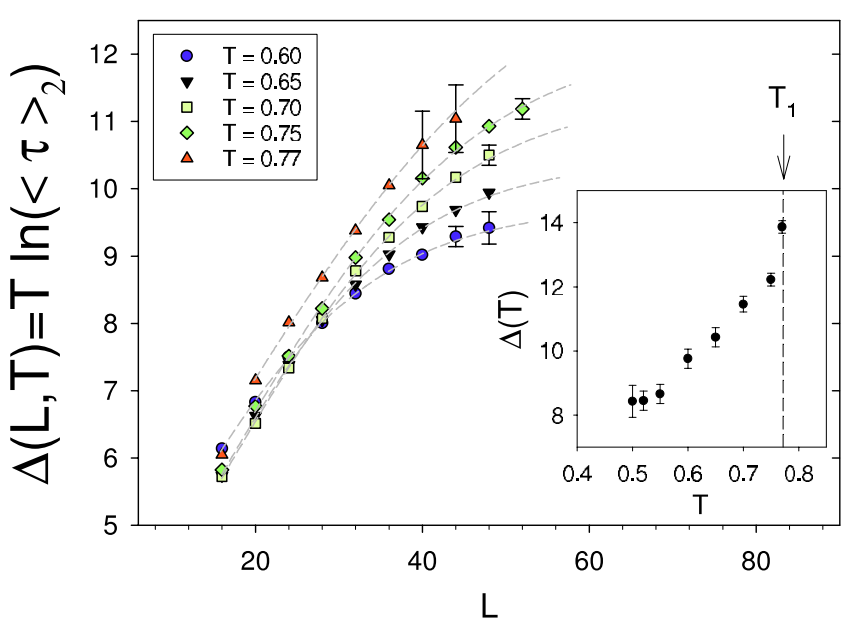

FIG. 6. (Color online) Estimated free energy barrier $\Delta(L, T)$ $\approx T \ln \left(\langle\tau\rangle_{2}\right)$ vs $L$ for different temperatures. The dashed lines correspond to a sigmoidal fitting. The inset shows the extrapolated free energy barrier $\Delta(T)=\Delta(\infty, T)$ vs $T$. The error bars are shown only when larger than the symbol size.

sarily equal to $\tau_{\text {nucl }}$, because after the creation of a critical droplet it follows a growth process until the whole system reaches the crystal phase (for the rather small system sizes we considered we can assume that there is only one critical droplet). Hence, $\langle\tau\rangle_{2}=\tau_{\text {nucl }}+\tau_{\text {growth. }}$. However, we can expect $\tau_{\text {growth }} \sim L^{a}$, so $\tau_{\text {nucl }} \gg \tau_{\text {growth }}$, at least as long as $L$ is large enough that we are in the scaling regime, but small enough so that the free energy barrier still depends on $L$. Under these conditions $\langle\tau\rangle_{2}$ give us a good approximation of $\tau_{\text {nucl }}$ and we can estimate $\Delta(L, T) \approx T \ln \langle\tau\rangle_{2}$ up to logarithmic corrections. Then, by fitting the curves of Fig. 6 using a sigmoidal function $\quad \Delta(L, T)=\Delta(T) /\left\{1+\exp \left[L^{*}(T)-L\right] / c(T)\right\} \quad\left[L^{*}(T)\right.$, $\Delta(T)$, and $c(T)$ are fitting parameters], we can extrapolate $\Delta(T)=\lim _{L \rightarrow \infty} \Delta(L, T)$. The extrapolated curve $\Delta(T)$ is shown in the inset of Fig. 6. We see that $\Delta(T)$ grows strongly as $T \rightarrow T_{1}^{-}$, while it appears to present a minimum around $T$ $=0.5$, or at least to saturate at a constant value as $T \rightarrow 0$. This is compatible with the results in [14] obtained from a different analysis where a divergent free energy barrier at the transition temperature $T_{1}$ was found in the thermodynamic limit. For quench temperatures $T<0.5$ very large relaxation times make it very difficult to obtain reliable numerical results. For future analysis we list in Table I the approximated fitted values of $\langle\tau\rangle_{2}$ for different values of $L$ and $T$.

We also calculated the relative probability of occurrence of nucleation and coarsening processes,

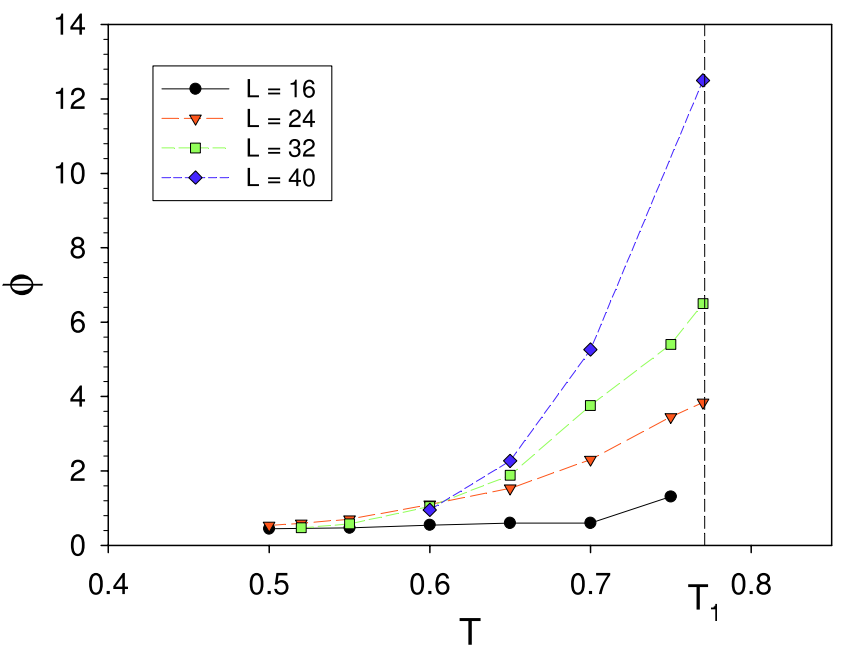

FIG. 7. (Color online) Relative probability Eq. (5) vs $T$ for $\delta$ $=2$ and different values of $L$.

$$
\phi(T) \equiv \frac{P_{\text {nucl }}}{P_{\text {coars }}}=\frac{\int_{-\infty}^{\infty} P_{2}(\ln \tau) d(\ln \tau)}{\int_{-\infty}^{\infty} P_{1}(\ln \tau) d(\ln \tau)}=\frac{\sigma_{2} A_{2}}{\sigma_{1} A_{1}},
$$

in the range $0.5 \leqslant T<T_{1}$. In Fig. 7 we see that $\phi(T)$ decreases monotonically with decreasing $T$. It seems to diverge as $T \rightarrow T_{1}^{-}$and $L \rightarrow \infty$, while becoming almost independent of $L$ for $T<0.6$, saturating in a constant value $\phi \approx 0.5$. This shows that nucleation dominates the relaxation near the melting point, while coarsening becomes dominant for deep quenches, although there is always a finite and relatively high $(\sim 1 / 3)$ probability of nucleation.

\section{RELAXATION AFTER A SLOW COOLING}

\section{A. Energy and structure factor}

We first analyze the behavior of the average energy per spin during a slow cooling; that is, we first equilibrate the system at some temperature $T_{0}>T_{2}$ and then decrease the temperature down to a final value $T_{f} \ll T_{1}$ according to the linear protocol $T(t)=T_{0}-r t$, where $r$ is the cooling rate, $t$ is in MCS, and the average is taken over several runs between $T_{0}$ and $T_{f}$.

In Fig. 8 we show an example. We see that below some value of $r$ the energy curve becomes almost independent of $r$.

In Fig. 9 we compare the cooling curves with the corresponding heating curve; this last curve is calculated by per-

TABLE I. $\langle\tau\rangle_{2}$ for different values of $L$ and $T$.

\begin{tabular}{lccccc}
\hline \hline \multicolumn{5}{c}{$T$} \\
\cline { 2 - 6 }$L$ & 0.60 & 0.65 & 0.70 & 0.75 & 0.77 \\
\hline 32 & $\sim 1.3 \times 10^{6}$ & $\sim 5.4 \times 10^{5}$ & $\sim 2.8 \times 10^{5}$ & $\sim 1.6 \times 10^{5}$ & $\sim 2.0 \times 10^{5}$ \\
36 & $\sim 2.4 \times 10^{6}$ & $\sim 1.1 \times 10^{6}$ & $\sim 5.7 \times 10^{5}$ & $\sim 3.3 \times 10^{5}$ & $\sim 4.7 \times 10^{5}$ \\
40 & $\sim 3.4 \times 10^{6}$ & $\sim 2.0 \times 10^{6}$ & $\sim 1.1 \times 10^{6}$ & $\sim 8.0 \times 10^{5}$ & $\sim 1.0 \times 10^{6}$ \\
44 & $\sim 5.3 \times 10^{6}$ & $\sim 3.0 \times 10^{6}$ & $\sim 2.0 \times 10^{6}$ & $\sim 1.4 \times 10^{6}$ & $\sim 1.6 \times 10^{6}$ \\
48 & $\sim 6.6 \times 10^{6}$ & $\sim 4.4 \times 10^{6}$ & $\sim 3.3 \times 10^{6}$ & $\sim 2.1 \times 10^{6}$ & \\
\hline \hline
\end{tabular}




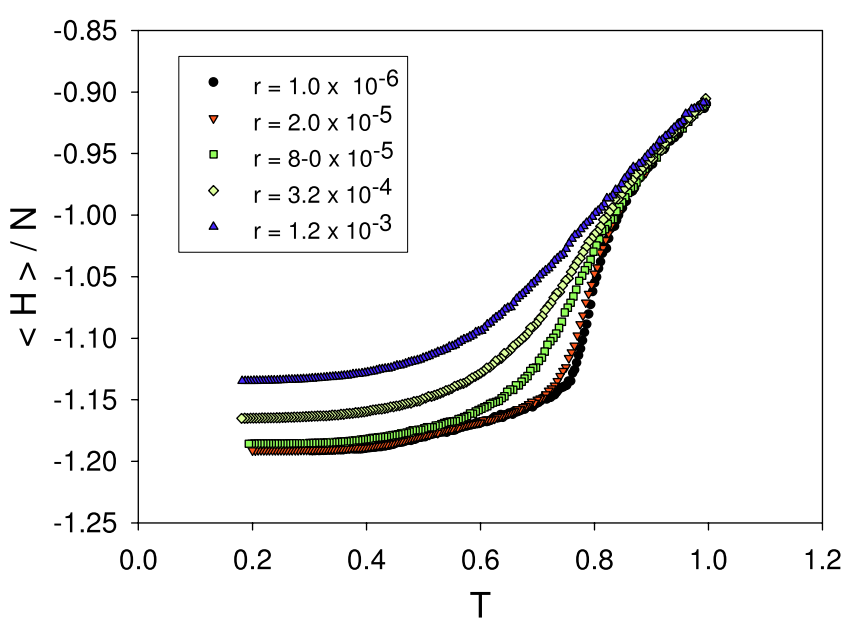

FIG. 8. (Color online) Average energy per spin in cooling from $T=0.9(\delta=2$ and $L=48)$ for different cooling rates (decreasing from top to bottom).

forming a linear heating from the ground state, starting at a very low temperature. We also compare the curves with the equilibrium values obtained in Ref. [14]. These results verify that (for this range of system sizes) metastability in the tetragonal-nematic transition is negligible, while there is a strong metastability associated with the stripe-nematic transition. It can be noted that the cooling curve turns down around $T=0.5$. From Table I we see that for $L \sim 50$ the nucleation time is $\tau_{\text {nucl }} \sim 4 \times 10^{6} \mathrm{MCS}$; then, for a cooling rate $r$ $=10^{-7}$, the time elapsed from the instant at which the temperature $T=T_{1}$ to the instant corresponding to a final temperature around $T=0.5$ will be $t=\Delta T / r \sim \tau_{\text {nucl }}$. Therefore, for that range of temperatures we would expect that in a large fraction of the realizations the system would have already equilibrated. Indeed, the results of Fig. 10 confirm this assumption. Moreover, the tracking of typical spin configurations for those realizations where the system did not equilibrate below $T=0.5$ shows that actually the system is no

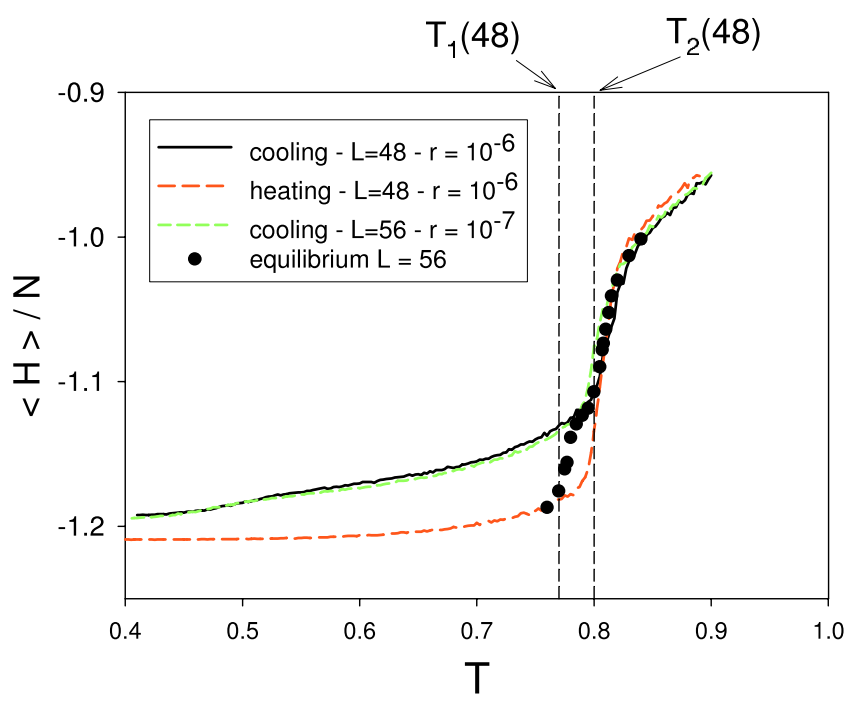

FIG. 9. (Color online) Average energy per spin cooling and heating curves ( $\delta=2$ and $L=48$ ).

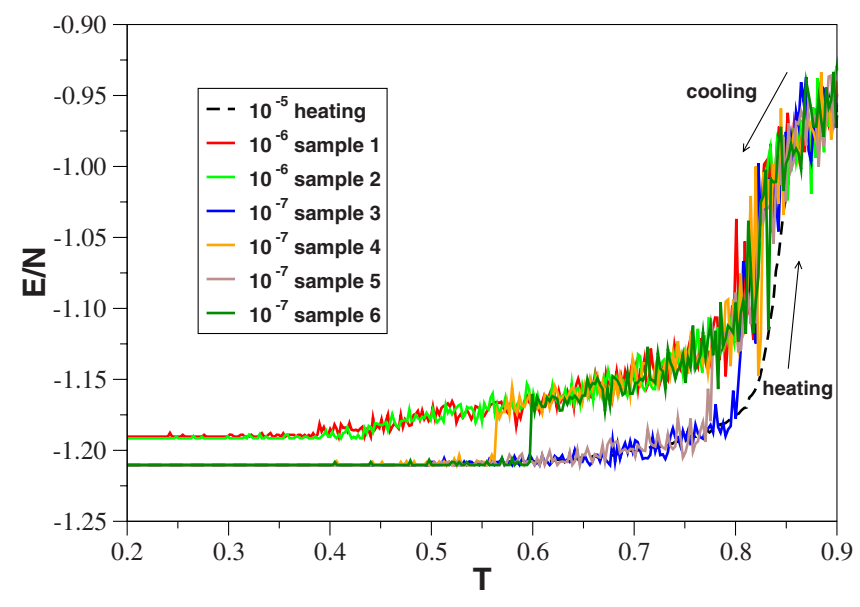

FIG. 10. (Color online) Energy per spin cooling curves: individual realizations ( $\delta=2$ and $L=48$ ).

longer in a nematic state; instead of that, it got stuck in another metastable state, with a mixture of stripes with widths $h=2$ and 3 . Actually the $h=3$ striped phase becomes metastable precisely for that range of temperatures [16].

In order to illustrate the qualitative differences between metastable nematic and the stable striped (smectic) states, we show in Fig. 11 two typical spin configurations obtained by heating and cooling at constant rate to the same temperature $T=0.77$ (close to $\left.T_{1}\right)$ for a rather large system size $(L$ $=120$ ). The main differences are due to the form of topological defects. While fluctuations in the striped state are characterized by a small density of coupled pairs of dislocations (mainly bridges), characteristic of a smectic state, strong fluctuations in the nematic give rise to a high density of unbounded dislocations. Such differences become more and more evident as the system size increases. The different kinds of topological defects give rise to different behaviors of the spatial correlations, or equivalently of the static structure factor (2). It was shown in Ref. [14] that in the nematic state for $\delta=2$ the structure factor presents two Lorentzian peaks along one of the coordinate directions, while it is almost zero anywhere else; the peaks are centered at $\pm k_{0}$, with $k_{0} \approx 1.48$. Moreover, it was shown that $S(\vec{k}) \sim L^{-1}$, showing that long-range order is completely suppressed in the ther- (a)

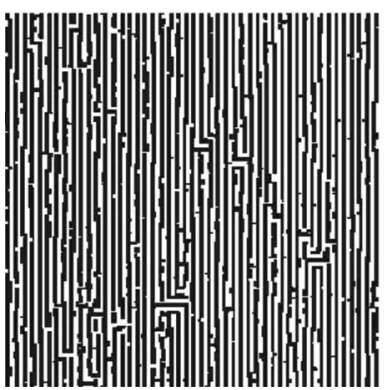

(b)

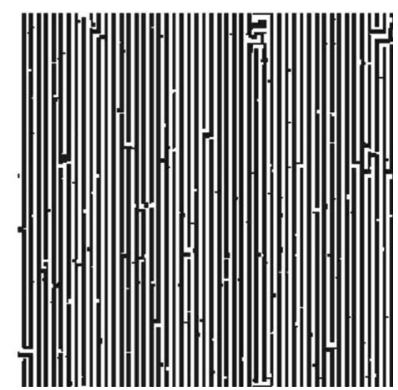

FIG. 11. Typical spin configurations for $\delta=2, T=0.77$, and $L$ $=120$, obtained by different thermal paths at constant rate $r=10^{-6}$. (a) Cooling from high temperature starting from a paramagnetic configuration. (b) Heating from the ground state. 


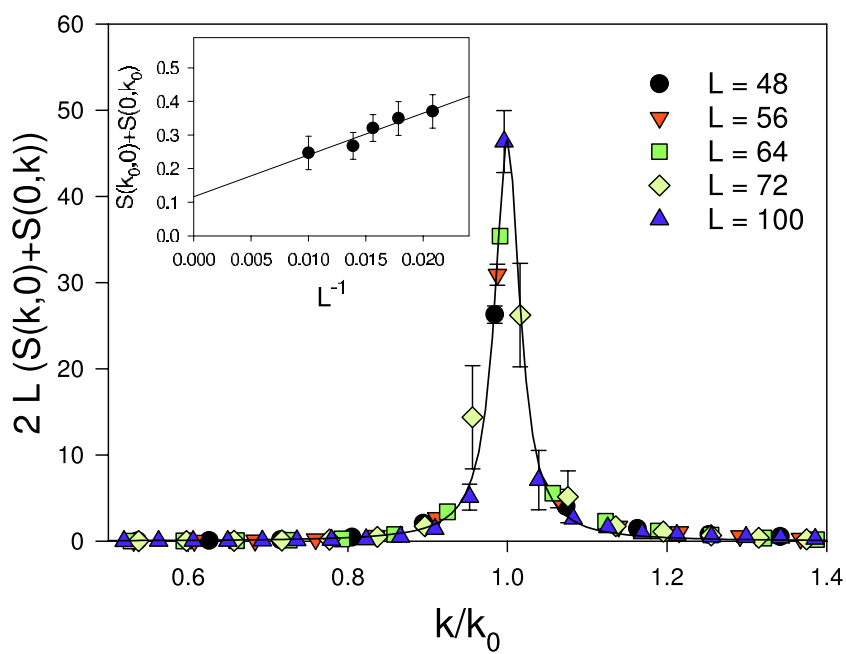

FIG. 12. (Color online) $2 L[S(k, 0)+S(0, k)]$ vs $k / k_{0}$ calculated after a cooling at constant rate $r=10^{-6}$ from high temperature down to $T=0.75$ for $\delta=2$ and different system sizes. The values of $k_{0}$ were obtained from Lorentzian fittings of either $S(k, 0)$ or $S(0, k)$ for every value of $L$. The continuous line corresponds to a Lorentzian fitting of the whole set of points. Error bars are only shown when larger than the symbol size.

modynamic limit [14]. On the other hand, in the striped state $S(\vec{k})$ presents two $\delta$ functions along one of the coordinate directions, centered at [14] $k= \pm \pi / 2$ (although in the smectic state a broadening of the peaks is expected, due to the existence of quasi-long-range order, for the present system sizes such broadening has not been detected). Following the same lines as in Ref. [14], we calculated the structure factor after a slow cooling down to $T=0.75<T_{1}$ for different system sizes. In all the cases the results could be well fitted by a superposition of two Lorentzian functions centered at $\pm k_{0}$ along one of the coordinate directions. In Fig. 12 we show $2 L[S(k, 0)+S(0, k)]$ vs $k / k_{0}$ for different system sizes. The data collapse into a single Lorentzian master curve in complete agreement with the expected behavior in a nematic state (see Ref. [14] for details).

\section{B. Correlations}

We now analyze the two-time correlation function

$$
C\left(t_{w}, t_{w}+t\right)=\frac{1}{N} \sum_{i}\left\langle\sigma_{i}\left(t_{w}\right) \sigma_{i}\left(t_{w}+t\right)\right\rangle
$$

after cooling from high temperature to a fixed temperature $T<T_{2}$. In this case we performed the cooling with a ladder protocol, that is, we reduced the temperature by steps of $\Delta T=0.01$, letting the system equilibrate during $10^{4} \mathrm{MCS}$ at each intermediate temperature. On the average, this corresponds to a cooling rate $r=10^{-6}$. Once we arrived to the measuring temperature (and after another $10^{4} \mathrm{MCS}$ ) we set $t=0$ and we saved the initial configuration. The autocorrelations (6) were then measured as a function of $t$ and $t_{w}$ and the whole curve was averaged over different realizations of the stochastic noise, starting always from the same initial configuration (this is to save CPU time). Some checks repeating

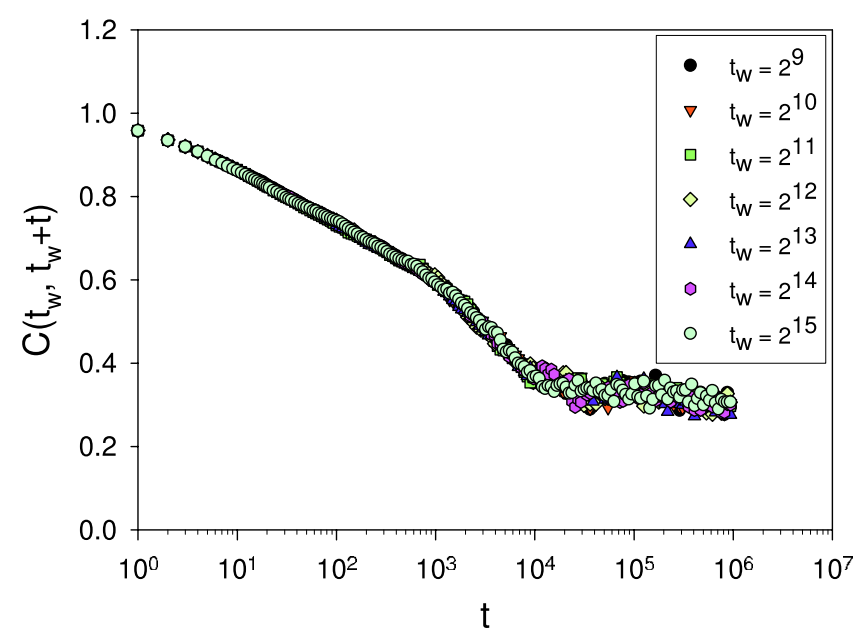

FIG. 13. (Color online) Two-time correlation function $C\left(t_{w}, t_{w}\right.$ $+t$ ) as a function of $t$ for $\delta=2, L=48$, and different waiting times $t_{w}$, after a quasistatic cooling at $T=0.78$. Sample size for averaging was 150 .

the calculation starting from different initial configurations (all obtained with the same cooling protocol) showed no difference from the previous one.

First of all we analyzed a cooling to a temperature $T_{1}(L)<T<T_{2}(L)$, which corresponds to the stable region of the nematic phase. From Fig. 13 we see that $C\left(t_{w}, t_{w}+t\right)$ $=C(t)$, as expected in a stable phase. We also noticed that the correlations do not decay to zero but to a plateau $C_{\text {plateau }}$ $\approx 0.37$. We will discuss the meaning of that plateau later.

In Fig. 14 we show the same calculation, but for a temperature $T<T_{1}(L)$. Both $t$ and $t_{w}$ were chosen such that $t$ $+t_{w} \ll \tau_{\text {nucl }}$. We see that again $C\left(t_{w}, t_{w}+t\right)=C(t)$, consistently with the expected quasistationary nature of a metastable state. We also see that the correlation also displays the characteristic plateau observed in the stable nematic state.

In Fig. 15 we show $C(t)$ for $T=0.7$ and $L=48$ in a very long run with $t$ up to $2 \times 10^{7}$ MCS. Comparing with Table I,

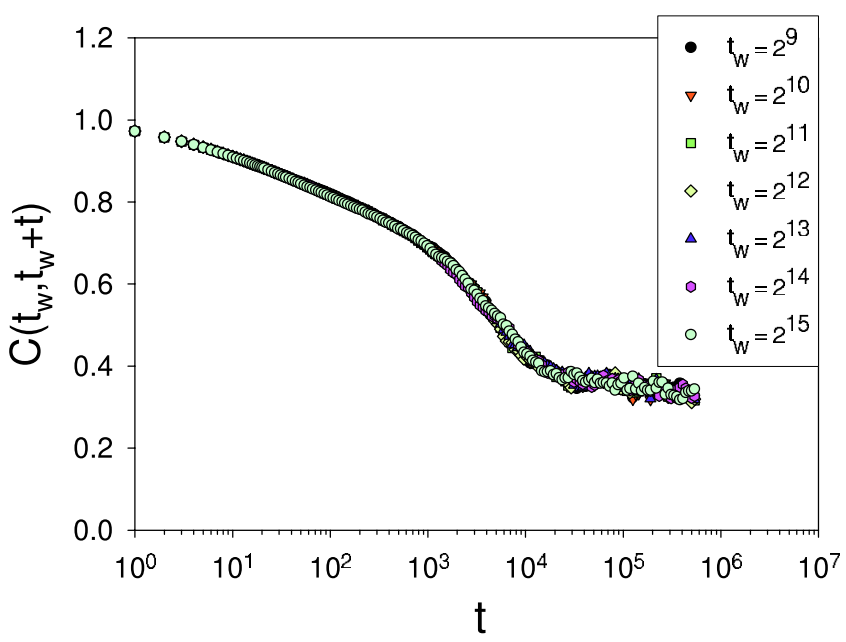

FIG. 14. (Color online) Two-time correlation function $C\left(t_{w}, t_{w}\right.$ $+t$ ) as a function of $t$ for $\delta=2, L=48$, and different waiting times $t_{w}$, after a quasistatic cooling at $T=0.7$. Sample size for averaging was 350 . 


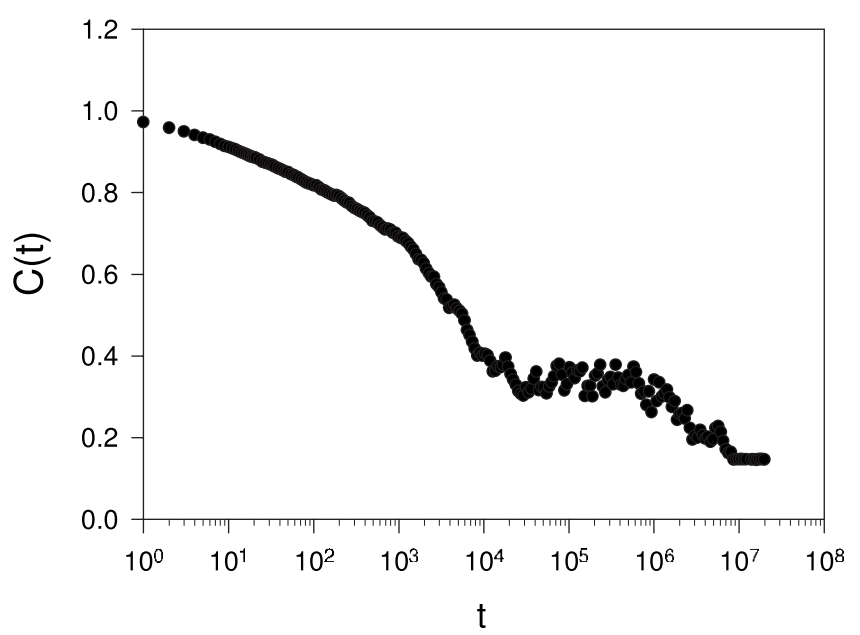

FIG. 15. Correlation function $C(t)$ as a function of $t$ for $\delta=2$, $L=48$, and different waiting times $t_{w}$, after a quasistatic cooling at $T=0.7$. Sample size for averaging was 80 .

we see that the second decay in the correlation coincides with the average nucleation time.

In Fig. 16 we show $C(t)$ for $L=36$ and different cooling temperatures. We see that the plateau appears for any temperature, but becomes higher in the nematic case.

What is the origin of the plateau? In principle one could argue that the problem is related to the study of the unconnected correlations instead of the connected ones which should filter out the mean value of the order parameter and leave only the fluctuations around it. In a nematic phase there is no positional order; in this respect the behavior in such a phase is like that in a paramagnetic one with $\langle s\rangle=0$. Then, in equilibrium, connected and unconnected correlations should be equal, both relaxing to zero at long times. What we have found is that this does not happen; we observe a plateau in a restricted time window. In Fig. 17 we show $C_{\text {plateau }}$ as a function of $L$ for different temperatures. For temperatures in the tetragonal liquid region, we see that for large enough sizes the plateau decays as $1 / \sqrt{N}$, as expected in a disordered state. However, for small sizes the plateau is independent of $L$ and the crossover size increases as the temperature approaches

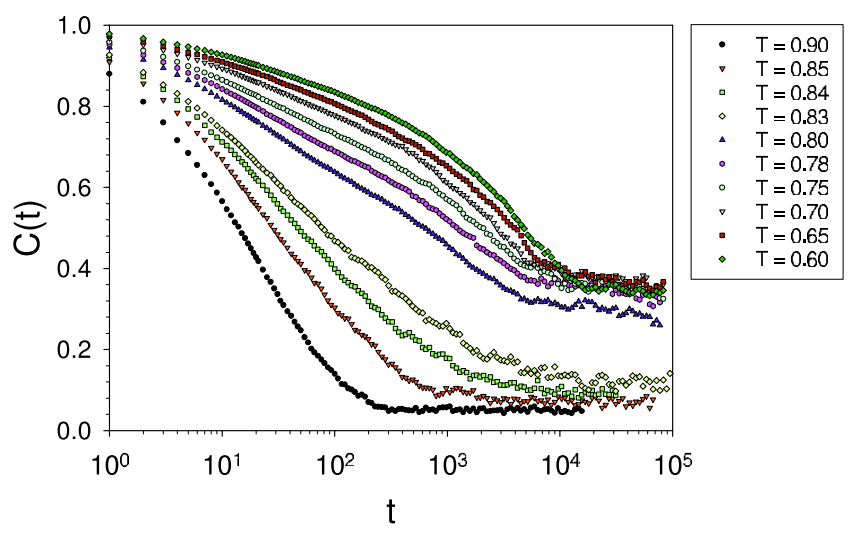

FIG. 16. (Color online) Correlation function $C(t)$ as a function of $t$ for $\delta=2$ and $L=36$ after a quasistatic cooling at different final temperatures (increasing from top to bottom).

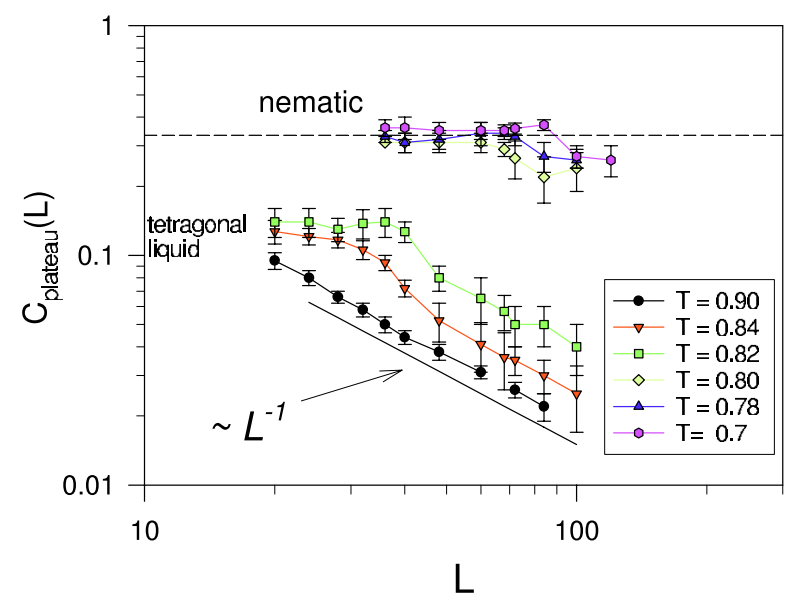

$1 / 3$

FIG. 17. (Color online) Correlation function plateau $C_{\text {plateau }}$ as a function of $L$ for $\delta=2$ and different temperatures.

$T_{2}$. Once we are in the nematic phase (either stable or metastable) the plateau for small sizes becomes independent of $T$ (at least for a range of temperatures where the relative probability of nucleation is high; see Fig. 7) and it is roughly equal to $1 / 3$. Since in a stationary state the unconnected correlations we are considering here behave as $C(t)$ $\sim(1 / N) \Sigma_{i}\left\langle\sigma_{i}\right\rangle^{2}$, this result shows that in the nematic state one-third of the spins remain in a frozen state for small system sizes. For the sizes studied, the plateau is similar to the one observed in glass-forming liquids above the glass transition: due to increasing freezing of degrees of freedom the relaxation shows two steps, a first one to a plateau known in the glass literature as the "nonergodicity parameter" and a final one in which the system decorrelates structurally. Still, the present situation is more subtle because, as exemplified in Fig. 15, when the system escapes the plateau and goes to the final decorrelation with the initial configuration, the transition to the stripe state intervenes and a second (and final) plateau is observed, corresponding to the residual correlation between the final (stripe) state and the initial (nematic) state.

Assuming then that the nematic state in a finite system is characterized by this residual correlation, we can define a normalized correlation function $C^{\prime}(t)$ as

$$
C^{\prime}(t) \equiv \frac{\left[C(t)-C_{\text {plateau }}\right]}{\left[C(1)-C_{\text {plateau }}\right]},
$$

where $C^{\prime}(t)=0$ characterizes the equilibrium nematic state (either stable or metastable). In Fig. 18 we see the typical behavior of $C^{\prime}(t)$ for different temperatures from above $T_{2}$ down to temperatures well below $T_{1}$. Also $C^{\prime}(t)$ exhibits different behaviors above and below $T_{2}$. For temperatures above $T_{2}$ the curves are very well fitted (see Fig. 18) by a modified stretched exponential function of the type

$$
f(t)=A t^{\omega} e^{-(t / \tau)^{\gamma}} .
$$

This fact is peculiar to the tetragonal liquid state: it does not exhibit exponential decay, even at relatively high temperatures. This behavior is observed also for $\delta=1$. For temperatures below $T_{1}, C^{\prime}(t)$ exhibits two different regimes, as can be appreciated more clearly in Fig. 19. At short times $C^{\prime}(t)$ is 


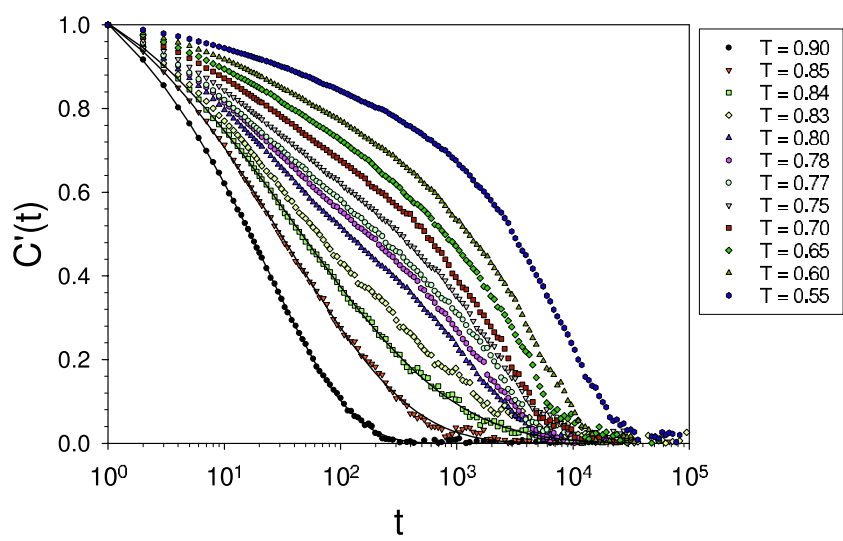

FIG. 18. (Color online) Normalized correlation function Eq. (7) as a function of $t$ for $\delta=2$ and $L=36$, after a quasistatic cooling at different temperatures (increasing from top to bottom). The continuous lines for temperatures above $T_{2}$ correspond to fittings using Eq. (8).

again well fitted by a function of the type Eq. (8). For times longer that some crossover time, $C^{\prime}(t)$ enters into a pure stretched exponential regime, i.e., the best fit is obtained by a function of the type

$$
f(t)=A e^{-(t / \tau)^{\gamma}}
$$

Finally, we analyzed the temperature dependence of the relaxation time $\tau$. Since there is too much uncertainty in fitting a curve with three parameters, instead of considering $\tau$ we defined another correlation time $\tau^{*}$ as the time at which $C^{\prime}\left(\tau^{*}\right)=0.1$. In Fig. 20 we compare the temperature dependence of $\tau^{*}$ in different scale representations and for different system sizes. In Fig. 20(a) we show an Arrhenius plot of $\tau^{*}$. The dashed lines in the figure correspond to fits using a Vogel-Fulcher-Tamman (VFT) form

$$
\tau^{*}=\tau_{0} \exp \left(\frac{A}{T-T_{0}}\right),
$$

which is usually associated with the behavior of relaxation

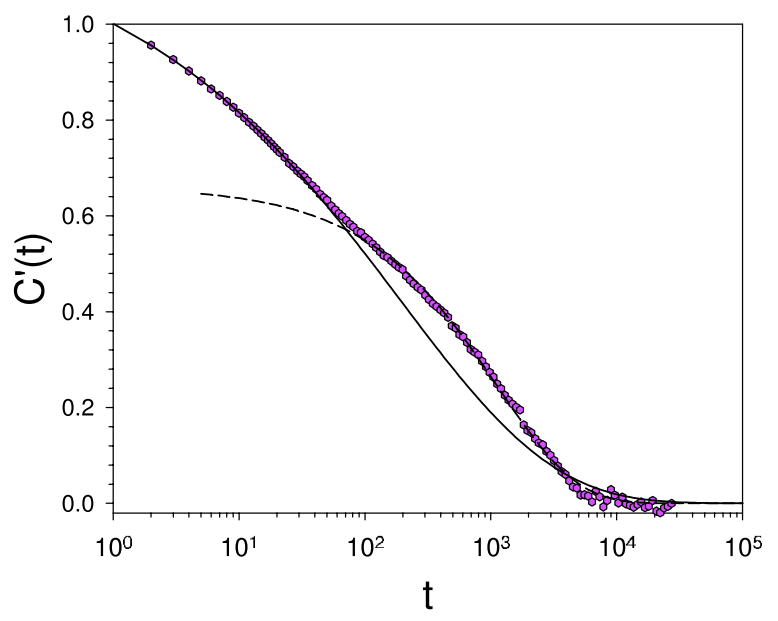

FIG. 19. (Color online) Normalized correlation function Eq. (7) as a function of $t$ for $\delta=2$ and $L=36$, after a quasistatic cooling at $T=0.78$. The continuous and dashed lines correspond to fittings using Eqs. (8) and (9) respectively.
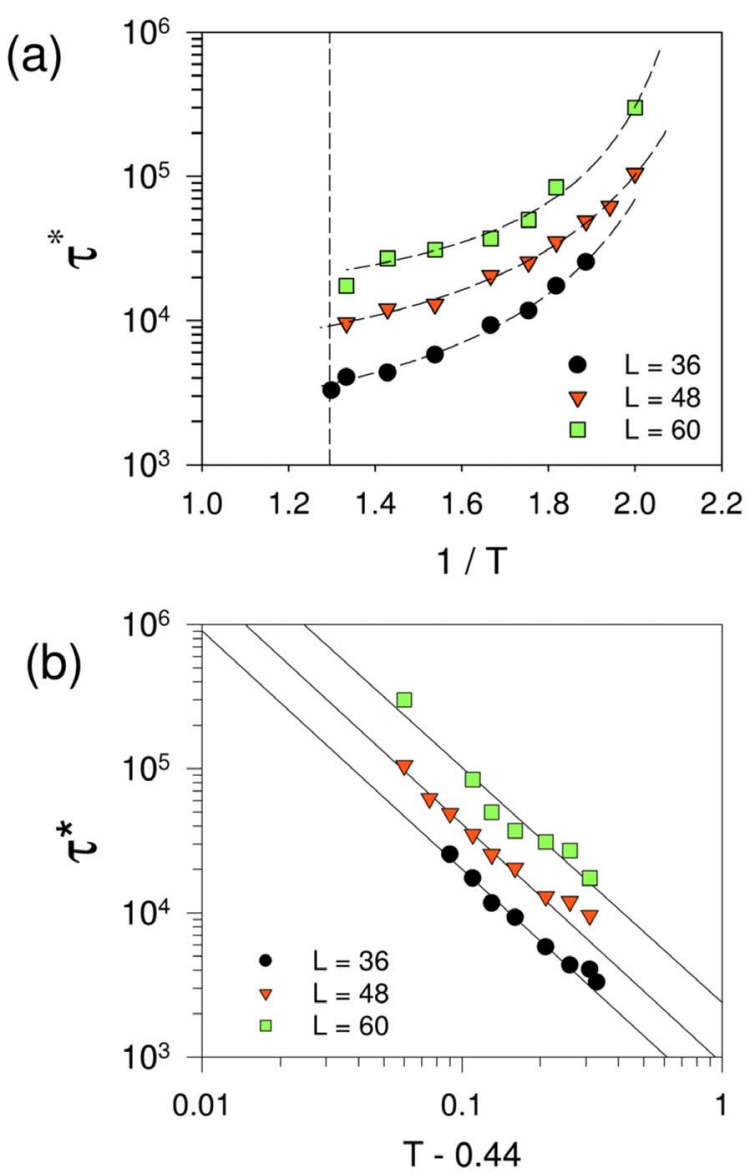

FIG. 20. (Color online) Relaxation time $\tau^{*}$ vs temperature for $\delta=2$ and different system sizes. (a) Arrhenius plot. The dashed lines correspond to fittings using the VFT form. The vertical line corresponds to $1 / T_{1}$. (b) $\log -\log$ plot of $\tau^{*}$ vs $T-0.44$. The full lines correspond to linear power law fittings with exponents $-1.65(L$ $=25$ and 48$)$ and $-1.63(L=60)$.

times in a fragile glass former, according to Angell's classification [24]. The fitting values of $T_{0}$ for the different system sizes are shown in Table II. We also show the fragility parameter $K \equiv T_{0} / A$, which show very high values around $K$ $\approx 1$. In Fig. 20 (b) we show the same results in a log-log plot. It is seen that they can also be fitted with a power law of the type $\tau^{*}=A\left|T-T_{0}\right|^{-b}$ with $T_{0}=0.44$; the obtained values $(b$ $=1.65 \pm 0.05$ for $L=36$ and $48 ; b=1.63 \pm 0.1$ for $L=60)$ are consistent with a single exponent independent of $L$. Although the best fitting is obtained with the VFT form Eq. (10), a power law fit cannot be discarded.

TABLE II. Fitting values of $T_{0}$ and the fragility parameter $K$ $\equiv T_{0} / A$ for the relaxation time $\tau^{*}$ as a function of the supercooling temperature using a VFT form.

\begin{tabular}{lcc}
\hline \hline$L$ & $T_{0}$ & $K$ \\
\hline 36 & $0.41 \pm 0.025$ & $1.20 \pm 0.4$ \\
48 & $0.40 \pm 0.015$ & $1.25 \pm 0.3$ \\
60 & $0.44 \pm 0.075$ & $2.10 \pm 0.8$ \\
\hline \hline
\end{tabular}




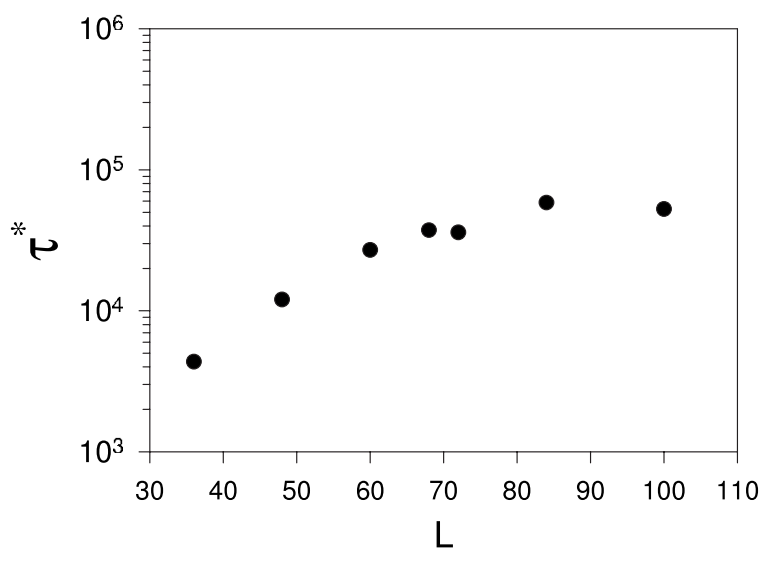

FIG. 21. Relaxation time $\tau^{*}$ vs $L$ for $\delta=2$ and $T=0.7$.

Notice the strong dependence of $\tau^{*}$ on $L$ for the range of sizes here considered. In Fig. 21 we show $\tau^{*}$ as a function of $L$ for a fixed temperature in the metastable region. We see that $\tau^{*}$ becomes independent of $L$ for sizes larger than $L$ $\sim 80$, which coincides with the crossover value observed for the plateau, suggesting the same origin for both finite-size effects.

\section{DISCUSSION AND CONCLUSIONS}

The main result of this work is the observation of interplay between nucleation and coarsening, a feature that, to our knowledge, has not been seen before. In other words, coarsening happens usually as a competition between domains of different stable phases. In the present case both direct coarsening of the stripe phase and nucleation of the same phase on a nematic background can happen with a probability that depends on the final temperature. Nucleation processes are associated with a strong metastability of the nematic phase. If the final temperature $T_{f}<T_{1}$ is not too far below the stripenematic transition temperature, the probability of nucleation is high: during the quench the system initially relaxes to the nematic phase, which is metastable below $T_{1}$ until finally it decays through nucleation to the equilibrium stripe phase. For final temperatures deep in the stripe phase, simple coarsening processes between stripe states with different orientations have larger probability of occurrence than nucleation. Although no detailed theory is available for predicting this behavior in the whole temperature range, classical arguments assuming homogeneous nucleation, coarsening with a nonconserved order parameter and finite-size scaling give a reasonably good interpretation of all the observed phenomenology. The origin of this interplay may rely on the fact that the stable and metastable phases share the same orientational symmetry, so we can have domains with stripes (or pieces of stripes) that can be oriented along the same directions of the square lattice substrate in both phases.

Quasiequilibrium autocorrelations in the stable and metastable nematic regions show a plateau whose nature is not completely clear. For temperatures where the nematic phase is only metastable, this plateau appears in a finite time window until a final decay for times $t \geqslant \tau_{\text {nucl }}$. For temperatures in the equilibrium nematic phase the behavior is more subtle. Although in a first approximation one could consider it to be a distinct characteristic of the nematic phase, in which a fraction of the spins remain practically frozen, we got indications that for the larger sizes the height of the plateau begins to diminish, signaling a possible finite-size origin. One possibility is that the fraction of frozen spins in the nematic phase diminishes with system size, in which case the plateau will eventually disappear for sizes large enough. More work is needed to clarify the behavior of equilibrium relaxations in large samples.

The present results also confirm the existence of the intermediate nematic phase, as observed in equilibrium simulations in $[14,16]$; all the dynamical observations are in agreement with the reported equilibrium results. In fact, a nematic phase with quasi-long-range order is expected to be generic in isotropic systems with competing interactions [25]. Recent analytic results on a continuous version of the present system show the presence of an isotropic-nematic transition which is in the Kosterlitz-Thouless universality class, while the stripe phase with true positional long-range order turns out to be unstable for continuous and isotropic systems in $d=2[25]$ in the thermodynamic limit, at variance with the discrete model studied here. Nevertheless, it is important to note that, although truly unstable in the thermodynamic limit, stripe domains grow already in the paramagnetic phase with decreasing temperature [26] and may be relevant for observations in finite-size systems, as in simulations for example. In the present case, the stability of the lowtemperature stripe phase may be due to the symmetry breaking effect of the lattice and the sharp nature of the Ising domain walls. A unified interpretation of the nature of the transitions in the discrete and continuous models is still lacking.

A final important point concerns the strong increase observed in the stationary relaxation time $\tau^{*}$ in the metastable nematic phase. Although a spinodal instability cannot be excluded, the stretched exponential relaxation and the super Arrhenius (or power law) behavior of $\tau^{*}(T)$ point to an increasing cooperative and glassy dynamics. Indeed, a very similar behavior in a related model, namely, the Coulomb frustrated model in three dimensions, has been interpreted as a evidence of fragile-glass-forming phenomenology [27]. Recent experimental results on $\mathrm{Fe}$ on $\mathrm{Cu}(100)$ ultrathin films [8] have suggested the possibility of a stripe-liquid to stripeglass transition in the vicinity of the stripe ordering temperature. Stripe-glassy behavior can be interpreted within the frustration-limited domain theory [28] or the random firstorder transition $[29,30]$ scenarios. We have not attempted a quantitative comparison with either theory, which remains an interesting subject for future work.

\section{ACKNOWLEDGMENTS}

This work was partially supported by grants from CONICET, FONCyT Grant No. PICT-2005 33305, SeCyTUniversidad Nacional de Córdoba (Argentina), CNPq, and CAPES (Brazil), and ICTP Grant No. NET-61 (Italy). 
[1] A. Hubert and R. Schafer, Magnetic Domains (SpringerVerlag, Berlin, 1998).

[2] P. Politi, Comments Condens. Matter Phys. 18, 191 (1998).

[3] A. Giuliani, J. L. Lebowitz, and E. H. Lieb, Phys. Rev. B 74, 064420 (2006).

[4] R. Allenspach, M. Stampanoni, and A. Bischof, Phys. Rev. Lett. 65, 3344 (1990).

[5] A. Vaterlaus, C. Stamm, U. Maier, M. G. Pini, P. Politi, and D. Pescia, Phys. Rev. Lett. 84, 2247 (2000).

[6] Y. Z. Wu, C. Won, A. Scholl, A. Doran, H. W. Zhao, X. F. Jin, and Z. Q. Qiu, Phys. Rev. Lett. 93, 117205 (2004).

[7] C. Won, Y. Z. Wu, J. Choi, W. Kim, A. Scholl, A. Doran, T. Owens, J. Wu, X. F. Jin, H. W. Zhao, and Z. Q. Qiu, Phys. Rev. B 71, 224429 (2005).

[8] O. Portmann, A. Vaterlaus, and D. Pescia, Phys. Rev. Lett. 96, 047212 (2006).

[9] Y. Yafet and E. M. Gyorgy, Phys. Rev. B 38, 9145 (1988).

[10] D. Pescia and V. L. Pokrovsky, Phys. Rev. Lett. 65, 2599 (1990).

[11] A. Abanov, V. Kalatsky, V. L. Pokrovsky, and W. M. Saslow, Phys. Rev. B 51, 1023 (1995).

[12] A. B. MacIsaac, J. P. Whitehead, M. C. Robinson, and K. De'Bell, Phys. Rev. B 51, 16033 (1995).

[13] S. A. Cannas, D. A. Stariolo, and F. A. Tamarit, Phys. Rev. B 69, 092409 (2004).

[14] S. A. Cannas, M. F. Michelon, D. A. Stariolo, and F. A. Tamarit, Phys. Rev. B 73, 184425 (2006).

[15] E. Rastelli, S. Regina, and A. Tassi, Phys. Rev. B 73, 144418 (2006).
[16] S. A. Pighin and S. A. Cannas, Phys. Rev. B 75, 224433 (2007).

[17] M. Carubelli, O. V. Billoni, S. Pighin, S. A. Cannas, D. A. Stariolo, and F. A. Tamarit, Phys. Rev. B 77, 134417 (2008).

[18] L. C. Sampaio, M. P. de Albuquerque, and F. S. de Menezes, Phys. Rev. B 54, 6465 (1996).

[19] J. H. Toloza, F. A. Tamarit, and S. A. Cannas, Phys. Rev. B 58, R8885 (1998).

[20] D. A. Stariolo and S. A. Cannas, Phys. Rev. B 60, 3013 (1999).

[21] P. M. Gleiser, F. A. Tamarit, S. A. Cannas, and M. A. Montemurro, Phys. Rev. B 68, 134401 (2003).

[22] I. Booth, A. B. MacIsaac, J. P. Whitehead, and K. De'Bell, Phys. Rev. Lett. 75, 950 (1995).

[23] J. Lee and J. M. Kosterlitz, Phys. Rev. B 43, 3265 (1991).

[24] C. A. Angell, K. L. Ngai, G. B. McKenna, P. F. McMillan, and S. W. Martin, J. Appl. Phys. 88, 3113 (2000).

[25] D. G. Barci and D. A. Stariolo, Phys. Rev. Lett. 98, 200604 (2007).

[26] R. Mulet and D. A. Stariolo, Phys. Rev. B 75, 064108 (2007).

[27] M. Grousson, G. Tarjus, and P. Viot, Phys. Rev. E 65, 065103(R) (2002).

[28] G. Tarjus, S. Kivelson, Z. Nussinov, and P. Viot, J. Phys.: Condens. Matter 17, R1143 (2005).

[29] J. Schmalian and P. G. Wolynes, Phys. Rev. Lett. 85, 836 (2000).

[30] H. Westfahl, J. Schmalian, and P. G. Wolynes, Phys. Rev. B 64, 174203 (2001). 